\title{
XXV Aniversario del Grupo de Trabajo de Endoscopia-Litiasis-Laparoscopia (LEL)
}

\author{
Vicente-Rodriguez J. \\ Fundació Puigvert. Barcelona. \\ Actas Urol Esp. 2008;32(8):773-778
}

\section{RESUMEN}

XXV ANIVERSARIO DEL GRUPO DE TRABAJO DE ENDOSCOPIA-LITIASIS-LAPAROSCOPIA (LEL)

Introducción: El objetivo de este artículo es relatar los avatares estructurales y cambios de contenido, vividos por nuestro Grupo de Trabajo, LEL durante veinticinco años.

Material y métodos: El material recopilado proviene de tres fuentes: las vivencias y experiencias del autor, la iconografía proporcionada por los coordinadores y, los datos obtenidos de programas, cartas, etc.

Este material ha sido cribado y ordenado en seis etapas y se ha procedido a señalar en cada una de ellas los datos específicos de cada jornada, los datos claves estructurales y los hechos más relevantes durante el periodo.

Resultados: Como consecuencia de la actividad del Grupo se han conseguido varios propósitos: protocolizar las técnicas endoscópicas del Aparato Urinario Inferior y prolongarlas al Aparato Urinario Superior: URS y cirugía percutánea, el tratamiento integral de la Litiasis, la defensa de técnicas propias (como la RTU frente alternativas) así como peritar las nuevas tecnologías (láser en Urología) y sustituir técnicas de cirugía abierta por otras pertenecientes al Grupo: NLP y Laparoscopia.

Conclusiones: Durante 25 años el Grupo de Trabajo LEL, se ha mantenido cohesionado y activo, participando en cambios internos estructurales y externos, motivados por la aparición durante este periodo, de espectaculares novedades: acceso endoscópico al Aparato Urinario Superior, tratamiento Integral de la Litiasis y alternativas a cirugías clásicas (Cirugía Percutánea, Cirugía Laparoscópica).

Palabras clave: Grupo de Trabajo. LEL. Litiasis. Endourología. Laparoscopia.

\section{ABSTRACT}

\section{ANNIVERSARY OF THE ENDOSCOPY-LITHIASIS-LAPAROSCOPY (LEL) WORKING GROUP}

Introduction: The purpose of this article is to describe the structural vicissitudes and content changes experienced by our Work Group, LEL over the last twenty-five years.

Materials and methods: The compiled material comes from three sources: the author's experiences; the iconography provided by the co-ordinators; and the data obtained from programmes, letters, etc.

This material has been analyzed and organized into six stages and the following has been highlighted in each of these: specific data from each day; key structural data; and the most relevant facts during the period.

Results: Various proposals have been made as a result of the Group's activity: to formally record the endoscopy techniques for the lower urinary tract and extend them to the upper urinary tract: URS and percutaneous surgery, the comprehensive treatment of lithiasis, the defence of our own techniques (such as UTR versus alternatives), as well as to provide an expert opinion on new technologies (laser in urology), and to replace open surgery techniques with others belonging to the Group: PNL and laparoscopy.

Conclusions: For 25 years, the LEL Work Group has stayed active and closely united. It has participated in both internal and external structural changes prompted by the appearance of spectacular new developments that have emerged over this period of time: endoscopic access to the upper urinary tract; comprehensive treatment of lithiasis; and alternatives to traditional surgeries (percutaneous surgery, laparoscopic surgery). 
$\mathrm{L}^{\circ}$ os grupos de trabajo, según consta en el reglamento de nuestra Asociación: "tienen como finalidad potenciar el desarrollo y profundizar en el conocimiento de las diversas facetas de nuestra Especialidad Urológica”. Con este precepto y reconociendo la importancia de la Endoscopia en la misma, se creó nuestro Grupo en Salamanca en 1984.

Constituido y organizado el Grupo Endourológico, a los cuatro años se amplia a paridad con el Grupo de Litiasis (Grupo L. E. 1988).

Durante algo más de una década este grupo bicéfalo, se estabiliza y se potencia, sumando conocimientos y desarrollando técnicas de cada una de las parcelas del grupo.

El nuevo siglo, se inicia con un intento fallido de diluir el Grupo y que responde potenciándose al incorporar la disciplina de Laparoscopia (Grupo L.E.L. 2000). Ésta adquiere un protagonismo casi excluyente, por lo que en la actualidad se han cambiado ciertas estructuras, para equilibrar sus tres componentes.

En paralelo han evolucionado los procedimientos, técnicas y hasta conceptos de las materias de nuestro Grupo: Ureterorrenoscopia, Cirugía Percutánea, desarrollo de utillaje, nuevas fuentes de energía, Litotricia Extracorpórea, técnicas laparóscopicas en sustitución de la cirugía abierta, cirugía robótica, etc.

La evolución en las estructuras y contenidos han obligado a sus coordinadores y componentes a adaptarse, sistematizar y reevaluar los espectacularmente rápidos cambios en Endoscopia, Litiasis y Laparoscopia.

Relatar estos cambios a lo largo de veinticinco años, es el objetivo de este trabajo.

\section{MATERIAL Y MÉTODOS}

El material recopilado se ha obtenido de tres fuentes principales: los recuerdos y vivencias personales del autor de este artículo; los datos iconográficos, actas, etc. proporcionados por la mayoría de los coordinadores y además, programas de jornadas, cartas, etc., cedidos por los propios coordinadores, recogidos por el autor o comunicados desde la Secretaría de la AEU.

Tan abundante información ha sido reducida, cribada y ordenada en seis etapas, señalando en cada una de ellas: la fecha, la sede, el presidente y el contenido de cada jornada. Asimismo, se adjuntan los coordinadores, "datos clave" en la evolución estructural y "hechos clave" acontecidos durante estos periodos en Endourología, Litiasis y Laparoscopia.

Esta variada e incompleta información dificulta relatar un verdadero artículo histórico de nuestro Grupo. Sin duda faltaran datos, hechos y colegas, por lo que solicito disculpas anticipadas.

\section{RESULTADOS}

Durante estos veinticinco años nuestro Grupo se ha mantenido unido, activo, innovador y autofinanciado, lo que nos ha permitido obtener excelentes resultados.

Es destacable que se haya logrado la sistematización, protocolización e innovaciones de las técnicas endoscópicas del Aparato Urinario Inferior y la prolongación de la Endo-urologi al Aparato Urinario Superior: Ureteroscopia y Cirugía Percutánea.

Tratamiento Integral de la Litiasis: suma de tratamiento medicamentoso (estudio metabólico, citratos), más técnicas endourológicas (URS y NLP con nuevas energías EHL, HoL, etc.) y la Litotricia Extracorpórea por Ondas de Choque (LEOC).

Defensa de técnicas propias (por ejemplo la RTU de HBP frente Alternativas), peritaje y crítica de nuevas tecnologías (por ejemplo Láser en Urología), y su utilidad en sustitución de otras clásicas: URS y NLP en Litiasis y TmsTUS y sobre todo la Cirugía Laparoscópica en sustitución de la Cirugía abierta: enucleación prostática, exeresis tumoral, reconstrucción funcional, etc.

Ha existido una interrelación positiva de nuestro Grupo con la Dirección de AEU y resto de Grupos. Por ejemplo los póster y Cursos de Adiestramiento de nuestras Jornadas han sido trasladadas a Congresos Nacionales y del mismo modo, pero en otra dirección, las Tutorías Laparoscópicas han necesitado del apoyo "nacional" para su promoción y docencia.

\section{DISCUSIÓN}

Como reseña resumida y ordenada, describiremos las seis etapas de los veinticinco años de nuestro Grupo, concluyendo con los hitos estructurales y científicos acontecidos durante este periodo (Tabla 1). 
Tabla 1

\begin{tabular}{lcccc}
\hline $1^{\text {a }}$ Etapa Grupo de Endoscopia (Fundacional). 1984 - 1988 \\
Coordinador: Dr. J. Vicente Rodriguez \\
\hline Año/Sede: & 1985 Madrid & 1986 Sevilla & 1987 Zaragoza & 1987 La Coruña \\
Presidente: & L.M. Ondina & M.García Pérez & G. Valdivia & M. González \\
Contenido: & $\begin{array}{l}\text { Temas y preguntas a demanda de C. Endoscópica (Aparáto Urinario } \\
\text { Sup. e Inf.) }\end{array}$ & & &
\end{tabular}

\section{"Endoscopia Total" \\ (Aparato Urinario Inferior y Superior)}

Datos clave

Durante el Congreso Nacional de 1984 en Salamanca, se aprueba y funda el Grupo de Trabajo de Endoscopia y como primer Coordinador se nombra al Dr. J. Vicente Rodriguez. Desde el inicio se asume el concepto de "Trabajo en Grupo" que se interpreta como reuniones interactivas, discusiones abiertas y preguntas a demandas.

Se realizan los Protocolos de Cirugía Endoscópica, aunque tuvieron escaso seguimiento (Dr. J Vicente 1987).

Las jornadas se intentan celebrar "adosados" o coincidentes con otros Cursos Urológicos (así sucedió en Zaragoza y La Coruña).

Se potencia la imagen utilizando la televisión en transmisión quirúrgica y la videografía Endoscópica ( $1^{\circ}$ y $2^{\circ}$ Curso Barcelona 1984 - 85. Dr. J. Vicente) como método de enseñanza de las técnicas quirúrgicas endoscópicas.

\section{Hechos clave}

Se alcanza y potencia la "Endoscopia Total":

Sistematización de la técnica del Aparato Urinario Inferior:

Adenoumectomía. Transuretral, incisión en "boca sonriente" del ureteroctele, et y se desarrolla y valora experimentalmente la resección con corriente bipolar (Dr. Vicente 1982, 1984 y 1987. Eur. Uol.)

Se refuerza la RTU de la HBP, a pesar y como reacción al trabajo de Roos en 1989, que al desacreditar esta técnica promueve la aparición de sus alternativas.

Diagnóstico y terapéuticas endourológicas del Aparato Urinario Superior: Ureteroscopia siguiendo la estela de su creador el Dr. E. Pérez-Castro y Cirugía Percutánea en Litiasis ( $1^{\mathrm{a}}$ NLP en España en Actas 1984. Dr. J. Vicente) y menos en tumores TUS, ambas técnicas siguen vigentes en la actuali- dad impulsadas por muchos componentes del Grupo (Dr. Banus, Dr. Arrabal, Dr. Hidalgo, Dr. Valdivia, Dr. Ibarlucea, etc.) (Tabla 2).

Tabla 2

$2^{\text {a }}$ Etapa ( $1^{\text {a }}$ L. E.): Grupo de Litiasis - Endoscopia 1988-1991 Coordinadores: Dr. J. Ruiz-Marcellan (Lts.) y Dr. E. Perez-Castro (End.)

\begin{tabular}{lcccc}
\hline Año/Sede: & 1988 Palma M. & 1989 San Juan & 1990 Salou & 1991 Valencia \\
Presidente: & M. Ozonas & A. Mira & J. Rey & F. Prosper \\
Contenido: & Comunicaciones:90 & Com. 44 M.R:1 & Com.=81 & Com.=76 Tesis=1 \\
& Video: 29. M.R=2 & Video: 16. Poster:6 & Video:15 M.R:2 & Video: 12. M.R:2
\end{tabular}

\section{Endourología y Litiasis = Patologias} Coincidentes, Técnicas Comunes

Datos clave

En 1988 se realiza la fusión de los grupos de Trabajo de Litiasis y Endoscopia (L.E.); ello requiere modificaciones, son necesarios dos coordinadores y dos días para que transcurran las jornadas anuales.

En estas jornadas se presentaron un mayor número de comunicaciones en Litiasis y mayor número de videos en Endoscopia; los datos corroboran esta afirmación, por ejemplo, en 1989 hubo 25 comunicaciones en Litiasis y 7 comunicaciones en Endoscopia; además también se realizaron 1 video de Litiasis y 15 vídeos en Endoscopia.

En esta etapa igualmente se inicia la presentación en forma de póster, y se organizan dos Mesas Redondas y la lectura de la mejor Tesis Doctoral en Litiasis.

Durante este periodo se potencian las terapéuticas comunes: por ejemplo: ureteroscopia = Litotricia in situ y Cirugía Percutánea: NLP.

También la aparición de la Litotricia Extracorpórea, supone un impacto que interfiere en el formato y actividad de ambos grupos.

\section{Hechos Clave:}

Irrupción de la Litotricia por Ondas de Choque en la Urología Nacional ( $1^{\mathrm{a}}$ en España por Dr. Ruíz Marcellan en 1984)

Modificación de la posición para Cirugía Percutánea renal: posición de decúbito supino del Dr. Valdivia (1990).

También destacan durante este periodo el impacto de las Alternativas iniciales de la RTU de HBP, inicio de utilización de láser en Urología: foto-coagulación en tumores, incisión en estenosis y empleo sustitutivo de prótesis prostáticas uretrales. 
En los tres se propagó su utilización clínica, hubo entusiasmos iniciales seguidos de una evaluación crítica posterior que suscitó desilusiones en la mayoría (Dr. J. Vicente, Dr. L. Resel, Dr. F. Jiménez, Dr. C. Rioja, etc.) (Tabla 3).

\section{Tabla 3}

$3^{\text {a Etapa. (2 }}{ }^{\text {a }}$ L. E.) Grupo de Litiasis Endoscopia 1993 -1996 Coordinadores: Dr. A. Conte (Lts). y Dr. C. Rioja (End.)

\begin{tabular}{ccccc}
\hline Año/Sede1 & 993 San Sebastián & 1994. Madrid & 1995. Formigal & 1996. Torremolinos \\
Presidente & J.A. López García & C. Hernánde & J. M. Ibarz & R. Burgos Rdez. \\
Contenido & Comunicación/Póster: 75 & C/Póster: 45 & Póster: 49 & Póster: 41 \\
& Vídeo: 28 M.R:2 & Vídeo: 16 M.R:2 & Vídeo: 28 M.R:2 & Vídeo:19 M.R:2 \\
& Premio Arregui & Conferencia 1 & Tec. Lovaco & Conf.3. \\
\hline
\end{tabular}

\section{Sólo póster, todos participan}

\section{Datos clave:}

Se instaura el "Sólo Póster" con lo que se consigue mayor número de participantes (generalmente los colegas más jóvenes) y mayor agilidad de presentación (más tiempo disponible). Se normaliza la inclusión de dos Mesas Redondas (una de Litiasis y una de Endoscopia) y se institucionaliza en el Programa de las Ponencias (por profesor nacional, extranjero y/o "no urólogo").

Se instaura el Premio Pedro Arregui, al mejor video y el Premio Robert, a la mejor Tesis Doctoral en Litiasis (la primera conseguida por el Dr. Conte). Durante esta etapa se inician los Cursos de "Laparoscopia en Cáceres".

\section{Hechos clave:}

Potenciación y simplificación del estudio metabólico en Litiasis (Dr. Conte, Dr. Grases).

Crítica inicial y opción selectiva de la LEOC; utilización de diferentes energías en Litotricia "in situ" (E. H., U. S., Neumática, Candela, Alexandrita, etc)

Opción selectiva de la Incisión Prostática en el Tratamiento de la HBP (Dr. Resel. 1993, Dr. Vicente. 1994 , etc) y crítica/abandono de varias alternativas a la RTU.

Inicio y desarrollo de la Cirugía Laparoscópicarenal (Dr. Valdivia, Dr. C. Rioja, Dr. C. Hernández).

Durante este periodo se da un gran impulso docente desde componentes del Grupo. Cabe resaltar el Tratado de Endourología (Dr. J. Vicente. 1996) y el seguimiento clínico del paciente litiasico (Dr. A. Conte. 1995).
Cursos de formación Laparoscópica en Cáceres. Realización y divulgación de técnicas específicas mediante vídeo-cirugía (Tabla 4).

\section{Tabla 4}

$4^{\text {a }}$ Etapa. (3 ${ }^{\text {a }}$ L. E.) Grupo de Litiasis Endoscopia 1997-2000

Coordinadores: Dr. M. Arrabal (Lts.) y Dr. C. Hernández (End.)

\begin{tabular}{ccccc}
\hline Año/Sede & 1997 Vall de Aran & 1998 Palma de G.C & 1999 Formigal & 2000 Granada \\
Presidente & C. Torrecilla & J. Ponce & M. González Martin & A. Zuloaǵa. \\
Contenido & Póster: 75 Vídeo: 33 & Póster: 58 Video:11 & Póster: 73 Vídeo: 18 & Póster: 87 Vídeo: 27 \\
& M.R:2 Taller de & M.R:2 Taller de & M.R:2 Taller de & M.R:2 Taller de \\
& Trabajo & Trabajo & Trabajo & Trabajo \\
\hline
\end{tabular}

Nuevas Técnicas: Cómo las hago y porqué las critico

Datos clave:

Estudios cooperativos a nivel nacional promovidos desde nuestro Grupo, Litotricia Extracorpórea en España; Congreso Nacional del 2000. Mesas Redondas celebradas en nuestras jornadas y vertidas para su publicación: en Actas Urológicas 2: 1015.1997 o como monografía: Litiasis Renal: estudio metabólico. 1999).

Durante este periodo se inicia en las reuniones del Grupo los “Talleres de Trabajo" "Cómo hago la Ureteroscopia” 1987, “"La Cirugía Percutánea" 1988 o "Según Casos Clínicos” 1999 - 2000) y se incluye en el contenido científico la "Valoración Crítica": ejemplo de esto último son las Alternativas a RTU de la HBP 1998 y Ondas de Choque 2000).

Hechos clave:

Introducción del citrato potásico en el Tratamiento de la Litiasis oxalocálcica (Dr. A. Rouseaud, P. Barceló. 1986).

Divulgación de la metodología básica en el estudio metabólico de la Litiasis (Dr. M. Arraba).

Impacto del Tratamiento Medicamentoso, más valoración crítica y descrédito de la mayoría de Alternativas en la HBP (Dr. R. Vela-Navarrete, Dr. Jiménez-Cruz, Dr. J. Vicente, etc.).

Difusión de la Cirugía Laparoscópica, desarrollo del instrumental/imagen y procedimientos de ayuda: Cirugía Laparoscópica mano-asistida (Dr. C. Hernández. 1997), Brazo Robotizado (Dr. A. Rosales 2000) (Tabla 5). 
Tabla 5

$5^{\text {a }}$ Etapa. (4 ${ }^{\mathrm{a}}$ L. E.) Grupo de Litiais Endoscopia 2001-2004 Coordinadores: Dr. A. Lancina (Lts.) y Dr. I. Fernández (End. Lap.)

\begin{tabular}{lcccc}
\hline Año/Sede & 2001 Andorra & 2002 Sigüenza & 2003 Gijón & 2004 Maspalomas \\
Presidente & J. Ruiz-Marcellan & J.M. Golvaño & V. Marua & J. Ponce \\
Contenido & Póster: 72 & Póster: 54 & Póster: 54 & Póster: 41 \\
& Víde0: 30 & Vídeo: 29 & Vídeo: 12 & Vídeo: 2 \\
& M.R:2 & M.R:2 & M.R:2 & M.R:2 \\
& Conf.1 T.T:1 & Conf. 1 T.T:1 & T. A: 1 & T. A: 1 \\
\hline
\end{tabular}

M.. . = Mesa Redonda T. T. $=$ Taller de Trabajo Conf. $=$ Conferencia T. A. = Taller de Adiestramiento

De la devaluación al refuerzo

Datos clave:

Intento fallido de la absorción/pérdida de autonomía del Grupo que resultó reforzado posteriormente con la suma de la Laparoscopia en 2004. El Grupo pasa a llamarse Grupo de Litiasis-Endourología-Laparoscopica (L.E.L) y a tener una página web propia (www.aeu.es/reunioneslel.es).

También se propone, no seguida, la creación dentro de la Urología, de una subespecialidad de Urolitiasis.

En este periodo se potencia la "enseñanza práctica” en las Reuniones: Talleres de Trabajo (Cómo hago...la RTU. Dr. J. Vicente 2001 y Ecografía Urología. Dr. I. Fernández 2002) y se inician los Talleres de Adiestramiento. (Ureteroscopia virtual, Dra. Laguna, Dr. Vicente 2003) y Sutura Laparoscopia (Dr. I. Fernández 2004).

\section{Hechos clave:}

Interés en divulgar los logros internos del Grupo: Guías médicas y campaña de prensa sobre litiasis (Dr. Lancina) y formación específica a los urólogos en general: dos libros del Dr. Lancina (Litogénesis Urinaria, enfermedades sistémicas y litiasis) y dos del Dr. Vicente (Tumores Vesicales Superficiales y HBP 2001) etc.

Durante este periodo se producen desarrollos espectaculares en utillaje endourológico/laparoscópico; ello facilita la reactivación del tratamiento con URS de litiasis (Hol.), estenosis, tumores, etc. (Dr. Valdivia, Dr. Amon, Dr. Boronat, etc.). Se prodigó la cirugía laparoscópica en patología tumoral: nefroureterectomía por TUS, prostatectomía radical, etc. (Dr. A. Rosales, Dr. L. MartínezPiñeiro, Dr. L. Ceccini, Dr. Amon, etc.) y se inicia la Nefrectomía Laparoscópica para transplante de vivo (Dr. A. Rosales, 2002) (Tabla 6).
Tabla 6

$6^{\text {a }}$ Etapa. (5 ${ }^{\text {a }}$ LEL) Grupo de Litiasis-EndoscopiaLaparoscopia 2005-2008

Coordinadores: Dr. JA. López-García (Lts.) y Dr. J. Amon (End. Lap.)

\begin{tabular}{lcccc}
\hline Año/Sede & 2005 Viella & El Escorial & 1995 Alicante & 1996. Murcia \\
Presidente & C. Torrecillas & I. Fernández & J. A. Galan & M. Pérez Albacete. \\
Contenido & Póster: 54 & Póster: 31 & Póster: 34 & Póster: 35 \\
& (14 Lap.) & (7 Lap.) & (12 Lap.) & \\
& Vide0: 48 & Vídeo: 32 & Video: 54 & Video: 50 \\
& (31 Lap.) & (26 Lap.) & (43 Lap.) & \\
C.P.: 1; & M.R: 2 & M.R: 2 & Nueva esctructura \\
& T.A: 2 S. P: 1 & Conferencia:1 & Conf.:1 T.A:1 S.P:1 & \\
\hline
\end{tabular}

Lap.: Laparoscopia C.P.: Curso Práctico T.A.: Taller de Adiestramiento S. P.: Sesión Provocativa

\section{Aniversario}

Datos clave

Rodaje de actividades sinérgicas y sumatorias de los tres contenidos del Grupo: Litiais, Endourología y Laparoscopia.

Enseñanza y aprendizaje de las técnicas de LEL mediante adiestramiento virtual: continuación de Talleres, simuladores, sedes estables (por ejemplo Urobionic en la Fundació Puigvert. 2004), potenciación de los cursos prácticos en las reuniones: "Cómo hago...”, Mesas Redondas de contenido común, sesiones provocativas, etc.

Propuesta y cambio de estructura de las Jornada, en vez de Conferencias y Mesas Redondas, realizar "Puntos de Técnica”, "Cara a Cara”, "Opiniones del Experto".

Se organiza a nivel nacional la Tutoría Laparoscópica como promoción, proyección y enseñanza de la Cirugía Laparoscópica Urológica (Dr. Amón).

Hechos clave

Aceptación/consenso de la utilidad de Laser Holmium en Litotricia in situ (Dr. López García, Dr. I. Fernández, Dr. F. Millán, Dr. Galán, Dr. Boronat, et al.).

Valoración e indicaciones quirúrgicas actualizadas de la HBP: RTU Bipolar, Láser KTP, enucleación con HoL (Dr. Valdivia, Dr. A. Santos, Dr. C. Hernández, Dr. Gómez Sancha, etc.)

En las jornadas: predominio de las técnicas laparoscópicas en detrimento de la Litiasis y Endourología; por ejemplo: los videos sobre Laparoscopia fueron el $64,5 \%$ en 2005 , 81\% en 2006 y $78 \%$ en 2007. Durante este periodo la Cirugía Laparoscópica se ve facilitada (¿sustituida?) por la Robótica ( $1^{\mathrm{a}}$ España. Dr. H. Villavicencio 2004). 


\section{COMPENDIO. CONCLUSIONES Hitos estructurales}

Reconocimiento de la importancia de la Endoscopia; se crea el Grupo en 1984. El principal tratamiento resolutivo de la Listiasis es Endoscópico. Fusión de los Grupos de Litiasis y Endoscopia (L.E. 1988).

Evolución y crecimiento: paso de "reunión de Grupo de Trabajo" a "mini-Congreso" (Reunión de otros Grupos. 1996).

Orientación progresiva en contenido "práctico": "Cómo hago...", Talleres de Trabajo, Aprendizaje Virtual, Reuniones Provocativas, etc.

Vocación permanente de enseñanza en nuestro Grupo: Libros, protocolos, videos, "Curso de Cáceres”, tutorías laparoscópicas, etc.

Tras intento de debilitar el Grupo éste se reafirma, estabiliza y potencia al incluir en el mismo la Laparoscopia (LEL 2004).

\section{Hitos cientificos}

Sistematización/racionalización de las técnicas endoscópicas del Aparato Urinario Inferior (Dr. J. Vicente).

Abordaje endoscópico del Aparato Urinario Superior: (URS Dr. E. Pérez Castro).

Cirugía endoscópica percutánea renal: tratamiento de litiasis, tumores, estenosis ostium (varias escuelas).

Litotricia Extracorpórea (LEOC, $1^{\mathrm{a}}$ en España, Dr. Ruíz-Marallan, 1984).

Citrato potásico en Litiasis oxalocálcica (Dr. A. Rousaud, 1986).
Nuevas fuentes de energía: US., Neumática, Lásers como aplicación en Litiasis, Endoscopia e inicial en Laparoscopia.

Innovación en imagen e instrumental: Videocirugía, miniaturización, flexibles, etc, que multiplican las aplicaciones clínicas.

Simplificación del estudio metabólico y test de riesgo litógeno (Dr. Grases 2004).

Avatares en el tratamiento endourológico de la HBP: RTU “en exclusiva”, decepción de las Alternativas hasta el Tratamiento integral: RTU uni/bipolar, vaporización KTP, enucleación HoL.

Irrupción, desarrollo y "vulgarización" de la Cirugía Laparoscópica gracias a instrumental específico, ayudas técnicas, Robótica, etc.

\section{ADENDUM}

Recuerdo emocionado a dos excelentes urólogos, amigos entrañables, que ya no pueden colaborar y prestigiar nuestro Grupo: Dr. Pedro Arregui (19521991) y Dr. Francisco Lovaco (1945-2007). Allá dónde estén, permanecen entre nosotros.

Correspondencia autor: Dr. J. Vicente-Rodríguez Servicio de Urología

Fundació Puigvert

Cartagena 340-350 - 08025 Barcelona

Tel.: 934169700

E-mail autor: jvicente@fundacio-puigvert.es

Información artículo: Original - Revisión histórica

Trabajo recibido: mayo 2008

Trabajo aceptado: junio 2008 